Pacific Journal of Mathematic 


\title{
NOTE ON SOME TAUBERIAN THEOREMS OF O. SZÁSZ
}

\author{
C. T. RA JAG OPAL
}

1. Introduction. The object of this note is to record extensions of the Tauberian theorems for Abel summability which form the subject of a recent paper of Szász in this journal [6, Theorem 2]. The extensions given as Theorems II, III', concern a process of summability which may be called the $(\Phi, \lambda)$--process, discussed elsewhere [3] and defined below. Theorems II, III' include similar results, given as Theorems I, III, which are implicit in [3].

The process of $(\Phi, \lambda)$-- summability is defined for any real series $\sum a_{n}$ as follows.

Let $\phi(u)$ satisfy the following conditions:

C (i) $\phi(u)$ is positive, continuous and monotonic decreasing in $(0, \infty)$;

C (ii) $\phi(0)=1, \int_{\epsilon}^{\infty}\{\phi(u) / u\} d u$ is convergent for every $\epsilon>0$;

C ( iii) $\phi(u)$ has a continuous derivative $-\psi(u)$ in $(0, \infty)$, this derivative being, on account of (i), negative and such that

$$
\phi(u)=\int_{u}^{\infty} \psi(x) d x
$$

C (iv) $\psi(u)$ is monotonic decreasing and has a continuous derivative in $(0, \infty)$;

$\mathrm{C}(\mathrm{v}) \quad \int_{0}^{\infty} u^{i x} \psi(u) d u \neq 0 \quad(-\infty<x<\infty)$.

Let

$$
\Phi_{\lambda}(t)=\sum_{n=1}^{\infty} a_{n} \phi\left(\lambda_{n} t\right), t>0,0=\lambda_{0}<\lambda_{1}<\lambda_{2}<\cdots, \lambda_{n} \rightarrow \infty .
$$

Then $\sum_{n=1}^{\infty} a_{n}$ is said to be $(\Phi, \lambda)$ - summable if

$$
\lim _{t \rightarrow+0} \Phi_{\lambda}(t) \quad \text { exists. }
$$


Examples of $\phi(u)$ which satisfy the conditions (C) are furnished by

$$
\begin{aligned}
& \phi(u)=e^{-u} \quad[\text { Abel-Laplace kernel], } \\
& \phi(u)=(1+u)^{-\rho}, \rho>0[\text { Stieltjes kerenel], } \\
& \phi(u)=u /\left(e^{u}-1\right), u>0, \phi(0)=1 \text { [Lambert kernel]. }
\end{aligned}
$$

The theorems of this note rest primarily on a result proved in my note already referred to [3, Theorem 2] and running as follows:

THEOREM A. Let $\phi(u)$ fulfil the conditions C (i)-(v). Suppose that $A(u)$ is a function of bounded variation in every finite interval of $(0, \infty), A(0)=0$. If

$$
\lim _{u \rightarrow \infty} \inf \frac{1}{u} \int_{0}^{u} u d\{A(u)\}>-\infty
$$

and

$$
\Phi(t)=\int_{0}^{\infty} \phi(u t) d\{A(u)\} \text { exists for } t>0 \text { and converges as } t \rightarrow+0,
$$

then we have

$$
\lim _{u \rightarrow \infty} \frac{A_{1}(u)}{u}=\lim _{t \rightarrow+0} \Phi(t), \text { where } A_{1}(u) \equiv \int_{0}^{u} A(x) d x
$$

2. Extensions of Szász's Tauberian theorems. It is clear that Theorem A can be restated for a $\lambda$-step function defined thus:

$$
A(u)=a_{1}+a_{2}+\cdots+a_{n} \text { for } \lambda_{n} \leq u<\lambda_{n+1} \text {, }
$$

$$
A(u)=0 \text { for } 0 \leq u<\lambda_{1} \text {. }
$$

For such a step function Szász has proved the following result [5, pp. 126127].

LEMmA 1. If $A(u)$ defined by (4) is $(R, \lambda, 1)$-summable to $s$, that is, if

$$
\frac{1}{x} \sum_{\lambda_{\nu \leq x}}\left(x-\lambda_{\nu}\right) a_{\nu} \equiv \frac{A_{1}(x)}{x} \rightarrow s \text { as } x \rightarrow \infty
$$

$A_{1}(x)$ being alternatively defined as in (3), and if, as $n \rightarrow \infty$, we have 


$$
\sum_{1}^{n}\left(\left|a_{\nu}\right|-a_{\nu}\right)^{p} \lambda_{\nu}^{p}\left(\lambda_{\nu}-\lambda_{\nu-1}\right)^{1-p}=O\left(\lambda_{n}\right) \quad(p>1)
$$

$$
\frac{\lambda_{n+1}}{\lambda_{n}} \rightarrow 1
$$

then $\sum_{n=1}^{\infty} a_{n}$ converges to $s$.

If, in Theorem A, $A(u)$ is the step function of (4), then (1) is a consequence of

$$
\lim _{n \rightarrow \infty} \inf \sum_{\nu=1}^{n} \frac{\lambda_{\nu}}{\lambda_{n}} a_{\nu}>-\infty
$$

which in turn is a consequence of the first condition in (5), as shown by Szász [5, p. 126], while (2) becomes the hypothesis of $(\Phi, \lambda)$-summability of $\sum a_{n}$. Thus Theorem A, in conjunction with Lemma 1, yields the following result.

THEOREM I. If $\sum_{n=1}^{\infty} a_{n}$ is $(\Phi, \lambda)$ - summable to $s$, then (5) implies that $\sum_{n=1}^{\infty} a_{n}$ is convergent to $s$.

Theorem I was proved by Szász in the case $\phi(u)=e^{-u}[5$, Theorem 4(a)]. It is the case $\lambda_{n}=n, \phi(u)=e^{-u}$ of this theorem which he has recently generalized [6, Theorem 2]. Repeating his arguments, in a slightly more general form, we obtain the following extension of Theorem I.

THEOREM II. If $\sum_{n=1}^{\infty} a_{n}$ is $(\Phi, \lambda)$-summable to $s$ and if, as $n \rightarrow \infty$, we have

$$
U_{n} \equiv \sum_{\nu=1}^{n} \lambda_{\nu}\left(\left|a_{\nu}\right|-a_{\nu}\right)=O\left(\lambda_{n}\right), \quad \lambda_{n+1} / \lambda_{n} \longrightarrow 1
$$

and

$$
\frac{U_{m}}{\lambda_{m}}-\frac{U_{n}}{\lambda_{n}} \longrightarrow 0 \text { whenever } \frac{\lambda_{m}}{\lambda_{n}} \rightarrow 1
$$

then $\sum_{n=1}^{\infty} a_{n}$ is convergent to $s$.

Theorem II is an extension of Theorem I in the sense that the hypotheses of the former cover those of the latter. This observation, contained in the next lemma, can be substantiated exactly as in its particular case for which $\lambda_{n}=n$ 
$[6, \mathrm{p} .119]$.

Lемма 2. The first relation in (5) implies the first relation in (6), while (5) as a whole implies (7) through the relation

$$
\frac{U_{m}-U_{n}}{\lambda_{n}} \rightarrow 0 \quad \text { as } \quad \frac{\lambda_{m}}{\lambda_{n}} \rightarrow 1 \quad(n \rightarrow \infty) .
$$

For the proof of Theorem II we require a further lemma which is virtually contained in Szász's proof of the case $\lambda_{n}=n, \phi(u)=e^{-u}$ of Theorem II.

LEMMA 3. If $\sum_{n=1}^{\infty} a_{n}$ is $(R, \lambda, 1)$ - summable to $s$, and the second condition of (6) holds along with (7), then $\sum_{n=1}^{\infty} a_{n}$ is convergent to $s$.

Proof. The hypothesis of $(R, \lambda, 1)$-summability of $\sum_{a_{n}}$ to $s$ implies that

$$
\sigma_{n} \equiv \frac{1}{\lambda_{n+1}} \sum_{\nu=1}^{n}\left(\lambda_{\nu+1}-\lambda_{\nu}\right) s_{\nu} \rightarrow s, \text { where } s_{n}=\sum_{\nu=1}^{n} a_{\nu}
$$

Now, we have the identities

$$
\begin{aligned}
& s_{n-1}-\sigma_{n+k}=\frac{\lambda_{n}}{\lambda_{n+k+1}-\lambda_{n}}\left(\sigma_{n+k}-\sigma_{n-1}\right) \\
& -\frac{1}{\lambda_{n+k+1}-\lambda_{n}} \cdot \sum_{\nu=0}^{k}\left(\lambda_{n+k+1}-\lambda_{n+\nu}\right) a_{n+\nu}, \\
& s_{n}-\sigma_{n-k-1}=\frac{\lambda_{n+1}}{\lambda_{n+1}-\lambda_{n-k}}\left(\sigma_{n}-\sigma_{n-k-1}\right) \\
& +\frac{1}{\lambda_{n+1}-\lambda_{n-k}} \cdot \sum_{\nu=0}^{k}\left(\lambda_{n-\nu}-\lambda_{n-k}\right) a_{n-\nu} \cdot
\end{aligned}
$$

By using these identities, exactly as they have been used by Szász in the case $\lambda_{n}=n[6, \mathrm{pp} .118-119, \mathrm{pp} .120-121]$, we can prove that

$$
\lim _{n \rightarrow \infty} \sup s_{n} \leq s, \lim _{n \rightarrow \infty} \inf s_{n} \geq s,
$$

whence the conclusion of Lemma 3 follows at once. 
Proof of Theorem II. From the first condition in (6) we get

$$
-\sum_{\nu=1}^{n} \lambda_{\nu} a_{\nu} \leq \sum_{\nu=1}^{n} \lambda_{\nu}\left(\left|a_{\nu}\right|-a_{\nu}\right) \equiv U_{n}=O\left(\lambda_{n}\right) .
$$

Therefore, defining $A(u)$ in Theorem A as the $\lambda$-step function of (4), we observe that hypothesis (1) of the theorem obtains as a result of $\left(1^{\prime}\right)$. Since hypothesis (2) also holds, in the form of the $(\Phi, \lambda)$-summability of $\sum a_{n}$, we are led to conclusion (3) in the form of the $(R, \lambda, 1)$-summability of $\sum a_{n}$. The desired conclusion now follows from Lemma 3 .

3. A second generalization of Theorem I. There is a generalization of Theorem I, different from Theorem II and very similar to a theorem of Delange [1, Theorème 8] which may be regarded as one more result of employing the technique of Szász embodied in Theorem $A$ and in certain arguments, already cited, involving ( $R, \lambda, 1)$-summability [5, pp. 126-127].

THEOREM I'. If $\sum_{n=1}^{\infty} a_{n}$ is a series $(\Phi, \lambda)$-summable to $s$, and if

(5) $\quad \sum_{1}^{n}\left(\left|a_{\nu}\right|-a_{\nu}\right)^{p} \lambda_{\nu}^{p}\left(\lambda_{\nu}-\lambda_{\nu-1}\right)^{1-p}=O\left(\lambda_{n}\right), \quad \quad p>1, n \rightarrow \infty$,

then $\left\{s_{n}\right\}$, the sequence of partial sums of $\sum_{n=1}^{\infty} a_{n}$, is such that

$$
\lim _{n \rightarrow \infty} \sup s_{n}=s, \quad \lim _{n \rightarrow \infty} \inf s_{n}=s-l
$$

where

$$
l=\lim _{n \rightarrow \infty} \sup \left(\left|a_{n}\right|-a_{n}\right) / 2
$$

The proof of Theorem I' is like that of Theorem I, but uses (in conjunction with Theorem A) the following lemma instead of Lemma 1.

Lemma $1^{\prime}$. If $\sum_{n=1}^{\infty} a_{n}$ is summable $(R, \lambda, 1)$ to $s$ and satisfies $\left(5^{\circ}\right)$, then the sequence $\left\{s_{n}\right\}$ of its partial sums behaves as in (8).

Proof. The first half of Szász's arguments [5, p. 126] proving Lemma 1, without any modification, establishes the first conclusion of (8).

To obtain the second conclusion of (8), we note that $l \geq 0$ by definition and $l<\infty$ by $\left(5^{\prime}\right)$ as shown in Lemma 4 which follows this proof. From the fact 


$$
a_{n} \geq-\frac{\left|a_{n}\right|-a_{n}}{2}
$$

we then infer that

$$
\lim _{n \rightarrow \infty} \inf a_{n} \geq-l
$$

We thereafter employ the second half of the aforesaid arguments of Szász [5, pp. 126-127] and reach the conclusion

$$
\lim _{n \rightarrow \infty} \inf s_{n} \geq s-l
$$

When $l=0$, (9) and the universal relation, $\lim$ inf $s_{n} \leq s$, together establish the second conclusion of (8). When $l>0$, we see that there is an increasing sequence of positive integers $n_{1}, n_{2}, \ldots, n_{r}, \ldots$ such that, as $r \rightarrow \infty,\left|a_{n_{r}}\right|-$ $a_{n_{r}} \rightarrow 2 l$ which implies (since $l>0$ ) that $a_{n_{r}}$ is ultimately negative and $a_{n_{r}} \rightarrow$ - $l$. Hence, when $l>0$,

$$
\begin{aligned}
\liminf _{n \rightarrow \infty} s_{n} \leq \limsup _{r \rightarrow \infty} s_{n_{r}} & =\lim _{r \rightarrow \infty} \sup \left(s_{n_{r}-1}+a_{n_{r}}\right) \\
& =\lim _{r \rightarrow \infty} \sup s_{n_{r}-1}-l \leq s-l
\end{aligned}
$$

Consequently, in the case $l>0$, the second conclusion of (8) follows from (9) and (10). The proof of Lemma $1^{\prime}$ is now complete.

Theorem $I$ is a special case of Theorem $I^{\prime}$ with $l=0$ as we can see from the following plain statement.

LEMMA 4. If $\sum_{n=1}^{\infty} a_{n}$ is any (real) series satisfying ( $\left.5^{\prime}\right)$, then

$$
\left|a_{n}\right|-a_{n}=O\left\{\left(\frac{\lambda_{n}-\lambda_{n-1}}{\lambda_{n}}\right)^{(p-1) / p}\right\}
$$

In particular, $\left(5^{\prime}\right)$ and the condition $\lim \lambda_{n} / \lambda_{n-1}=1$ together imply that $l \equiv \lim \sup \left(\left|a_{n}\right|-a_{n}\right) / 2=0$.

Now, in any (real) series $\sum a_{n}$,

$$
-\frac{\left|a_{n}\right|-a_{n}}{2}=\min \left(0, a_{n}\right)
$$


so that $\lim \inf a_{n} \geq 0$ implies $l=0$ and conversely. Thus, from Theorem I', we can say that there is a variant of Theorem I with the second condition of (5) replaced by $\lim$ inf $a_{n} \geq 0$; in fact, we can say more as follows.

For series $\sum_{n=1}^{\infty} a_{n}$ summable $(\Phi, \lambda)$ and satisfying the Tauberian condition $\left(5^{\prime}\right)$, a necessary and sufficient convergence condition is $\lim \inf a_{n} \geq 0$.

In the above statement, $\left(5^{\prime}\right)$ can be replaced by the following simpler condition which implies $\left(5^{\prime}\right)$ :

$$
\lim _{n \rightarrow \infty} \inf \frac{a_{n} \lambda_{n}}{\lambda_{n}-\lambda_{n-1}}>-\infty .
$$

4. A generalization of Theorem II. Following Szász, we have seen that conditions (6) and (7) of Theorem II together include the corresponding condition (5) of Theorem I. Following Szász further [6, $\$ 4.5]$, we see that conditions (6) and (7) together can be expressed in the Schmidt form, and the following equivalent of Theorem II obtained.

ТНЕОВЕМ III. If $\sum_{n=1}^{\infty} a_{n}$ is $(\Phi, \lambda)$-summable to $s$ and if, as $n \rightarrow \infty$,

$$
\frac{\lambda_{n+1}}{\lambda_{n}} \rightarrow \infty, \quad \sum_{\nu=n+1}^{m}\left(\left|a_{\nu}\right|-a_{\nu}\right) \longrightarrow 0 \quad \text { whenever } \frac{\lambda_{m}}{\lambda_{n}} \rightarrow 1,
$$

then $\sum_{n=1}^{\infty} a_{n}$ is convergent to sum s.

A generalization of Theorem III, related to it as Theorem $\mathrm{I}^{\prime}$ is to Theorem I, may now be stated in a familiar form as under.

THEOREM III'. If $\sum_{n=1}^{\infty} a_{n}$ is a series $(\Phi, \lambda)$-summable to $s$ and such that

$$
\text { either } \lim _{\delta \rightarrow+0} \lim _{n \rightarrow \infty} \sup \sum_{\lambda_{n+1} \leq \lambda_{\nu} \leq \lambda_{m}<(1+\delta) \lambda_{n}}\left(\left|a_{\nu}\right|-a_{\nu}\right)=0,
$$

$$
\text { or } \quad \lim _{\delta \rightarrow+0} \lim _{n \rightarrow \infty} \inf \underset{\lambda_{n+1} \leq \lambda_{\nu} \leq \lambda_{m}<(1+\delta) \lambda_{n}}{\min } \sum a_{\nu} \geq 0,
$$

then the sequence $\left\{s_{n}\right\}$ of partial sums of the series satisfies (8), provided that

$$
l \equiv \lim _{n \rightarrow \infty} \sup \left(\left|a_{n}\right|-a_{n}\right) / 2<\infty .
$$

Since $-a_{\nu} \leq\left|a_{\nu}\right|-a_{\nu}$, the first alternative of (12) implies the second, and the second is the only alternative that need be considered. Now the second 
alternative clearly implies that, on the assumption $\lambda_{n+1} / \lambda_{n} \rightarrow 1$, we have $\lim \inf a_{n} \geq 0$ and hence $l=0$. Therefore, in Theorem III', we can drop the explicit assumption $l<\infty$ and assume instead, either

$$
\lim \lambda_{n+1} / \lambda_{n}=1, \quad \text { or } \lim \inf a_{n} \geq 0 \text {, }
$$

getting the two cases of $l=0$ in the following corollary of which the first case is Theorem III.

COROLLARY III'. If a series $\sum_{n=1}^{\infty} a_{n}$ is $(\Phi, \lambda)$-summable and such that (12) holds along with

either $\lim \lambda_{n+1} / \lambda_{n}=1, \quad$ or $\quad \lim \inf a_{n} \geq 0$,

then $\sum_{n=1}^{\infty} a_{n}$ is convergent to $s$.

Corollary III', in the case $\phi(u)=e^{-u}$, is a classic theorem of Szász [4, p. 338]. Theorem III $^{\circ}$ in the same case and in an incomplete form has been already obtained by me [2, Theorem $F]$; its proof readily suggests the following

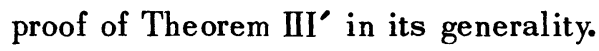

Proof of Theorem III'. Confining ourselves, as we may, to the second alternative of (12), we can show that this alternative and the condition $l<\infty$ (equivalent to $\left.\lim \inf a_{n}>-\infty\right)$ together imply ( $\left.1^{\prime}\right)$, using an argument indicated elsewhere [2, Lemma 1]. After this we can appeal to Theorem A.and infer that $\sum a_{n}$ is $(R, \lambda, 1)$-summable to $s$. The subsequent completion of the proof is along the lines of the proof of Lemma $1^{\prime}$.

\section{REFERENCES}

1. H. Delange, Sur les théorèmes inverses des procédés de sommation des séries divergentes (Deuxième Mémoire), Ann. Sci. École Norm. Sup. (3), 67 (1950), 199-242.

2. C. T. Rajagopal, On some extensions of Ananda Rau's converse of Abel's theorem, J. London Math. Soc., 23 (1948), $38-44$.

3. - A note on generalized Tauberian theorems, Proc. Amer. Math. Soc., 2 (1951), 335-349.

4. O. Szász, Verallgemeinerung und neurer Beweis einiger Sätze Tauberscher Art, Sitzungsber. Bayer. Akad. Wiss., Naturw. Abt. München, 59 (1929), 325 - 34.0.

5. - Converse theorems of summability for Dirichlet series, Trans. Amer. Math. Soc., 39(1936), 117-130.

6. , On a Tauberian theorem for Abel summability, Pacific J. Math., 1(1951), $117-125$.

Ramanujan Institute of Mathematics

MADRAS, IN DIA 


\title{
PACIFIC JOURNAL OF MATHEMATICS
}

\section{EDITORS}

\section{R. M. RobINSON \\ University of California \\ Berkeley 4, California}

\author{
*R. P. Dilwor TH \\ California Institute of Technology \\ Pasadena 4, California
}

\author{
E. F. BECKENBACH, Managing Editor \\ University of California \\ Los Angeles 24, California
}

${ }^{*}$ During the absence of Herbert Busemann in 1952.

\section{ASSOCIATE EDITORS}

$\begin{array}{llll}\text { R. P. DILWORTH } & \text { P. R. HALMOS } & \text { B } \emptyset \text { RGE JESSEN } & \text { J. J. STOKER } \\ \text { HERBERT FEDERER } & \text { HEINZ HOPF } & \text { PAUL LÉVY } & \text { E. G. STRAUS } \\ \text { MARSHALL HALL } & \text { R. D. JAMES } & \text { GEORGE PÓLYA } & \text { KÖSAKU YOSIDA }\end{array}$

\section{SPONSORS}

UNIVERSITY OF BRITISH COLUMBIA

CALIFORNIA INSTITUTE OF TECHNOLOGY

U NIVERSITY OF CALIFORNIA, BERKELEY

UNIVERSITY OF CALIFORNIA, DAVIS

UNIVERSITY OF CALIFORNIA, LOS ANGELES

UNIVERSITY OF CALIFORNIA, SANTA BARBARA

OREGON STATE COLLEGE

UNIVERSITY OF OREGON

\author{
UNIVERSITY OF SOUTHERN CALIFORNIA \\ STANFORD UNIVERSITY \\ WASHINGTON STATE COLLEGE \\ UNIVERSITY OF WASHINGTON \\ AMERICAN MATHEMATICAL SOCIETY \\ NATIONAL BUREAU OF STANDARDS, \\ INSTITUTE FOR NUMERICAL ANALYSIS
}

Mathematical papers intended for publication in the Pacific Journal of Miathematics should be typewritten (double spaced), and the author should keep a complete copy. Manuscripts may be sent to any of the editors. All other communications to the editors should be addressed to the managing editor, E. F. Beckenbach, at the address given above.

Authors are entitled to receive 100 free reprints of their published papers and may obtain additional copies at cost.

The Pacific Journal of Mathematics is published quarterly, in March, June, September, and December, by the University of California, Berkeley 4, California. The price per volume (4 numbers) is $\$ 8.00$; single issues, $\$ 2.50$. Special price to individual faculty members of supporting institutions and to individual members of the American Mathematical Society: $\$ 4.00$ per volume; single issues, $\$ 1.25$.

Subscriptions, orders for back numbers, and changes of address should be sent to the publishers, University of California Press, Berkeley 4, California.

Printed at Ann Arbor, Michigan. Entered as second class matter at the Post Office, Berkeley, California.

\section{UNIVERSITY OF CALIFORNIA PRESS • BERKELEY AND LOS ANGELES}




\section{Pacific Journal of Mathematics}

\section{Vol. 2, No. $3 \quad$ March, 1952}

Lars V. Ahlfors, Remarks on the Neumann-Poincaré integral equation .... 271

Leonard P. Burton, Oscillation theorems for the solutions of linear, nonhomogeneous, second-order differential systems ............ 281

Paul Civin, Multiplicative closure and the Walsh functions . . . . . . . . . . . 291

James Michael Gardner Fell and Alfred Tarski, On algebras whose factor algebras are Boolean .................................. 297

Paul Joseph Kelly and Lowell J. Paige, Symmetric perpendicularity in Hilbert geometries .................................. 319

G. Kurepa, On a characteristic property of finite sets .............. 323

Joseph Lehner, A diophantine property of the Fuchsian groups ......... 327

Donald Alan Norton, Groups of orthogonal row-latin squares ........... 335

R. S. Phillips, On the generation of semigroups of linear operators ....... 343

G. Piranian, Uniformly accessible Jordan curves through large sets of relative harmonic measure zero ........................ 371

C. T. Rajagopal, Note on some Tauberian theorems of $O . S z \tilde{A} ; s z \ldots \ldots \ldots 377$

Halsey Lawrence Royden, Jr., A modification of the Neumann-Poincaré method for multiply connected regions .................... 385

George H. Seifert, A third order irregular boundary value problem and the associated series ...................................... 395

Herbert E. Vaughan, Well-ordered subsets and maximal members of ordered sets....

Hans F. Weinberger, An optimum problem in the Weinstein method for eigenvalues.

Shigeki Yano, Note on Fourier analysis. XXXI. Cesàro summability of Fourier series. 